\title{
Monitoring the Dynamics of Monomer Exchange Using Electrospray Mass Spectrometry: The Case of the Dimeric Glucosamine-6-Phosphate Synthase
}

\author{
Guillaume Chevreux, ${ }^{1,3}$ Cédric Atmanene, ${ }^{1}$ Philippe Lopez, ${ }^{2}$ Jamal Ouazzani, ${ }^{2}$ \\ Alain Van Dorsselaer, ${ }^{1}$ Bernard Badet, ${ }^{2}$ Marie-Ange Badet-Denisot, ${ }^{2}$ \\ Sarah Sanglier-Cianférani ${ }^{1,4}$ \\ ${ }^{1}$ Laboratoire de Spectrométrie de Masse BioOrganique (LSMBO), Université de Strasbourg, IPHC, 25 rue Becquerel 67087 \\ Strasbourg, France \\ ${ }^{2}$ Centre de Recherches de Gif, ICSN-CNRS, Gif-sur-Yvette, France \\ ${ }^{3}$ Present Address: LFB, Structure and Glycobiology Laboratory, 3, avenue des Tropiques, Les Ulis, 91958, Courtabouf Cedex, France \\ ${ }^{4}$ CNRS, UMR7178, 67037 Strasbourg, France
}

\begin{abstract}
Escherichia coli glucosamine-6-phosphate synthase (GImS) is a dimeric enzyme from the glutamine-dependent amidotransferases family, which catalyses the conversion of D-fructose-6phosphate (Fru6P) and glutamine (Gln) into D-glucosamine-6-phosphate (GlcN6P) and glutamate, respectively. Extensive X-ray crystallography investigations have been reported, highlighting the importance of the dimeric association to form the sugar active site as well as significant conformational changes of the protein upon substrate and product binding. In the present work, an approach based on time-resolved noncovalent mass spectrometry has been developed to study the dynamics of GlmS subunit exchange. Using ${ }^{14} \mathrm{~N}$ versus ${ }^{15} \mathrm{~N}$ labeled proteins, the kinetics of GlmS subunit exchange was monitored with the wild-type enzyme in the presence of different substrates and products as well as with the protein bearing a key amino acid mutation specially designed to weaken the dimer interface. Determination of rate constants of subunit exchange revealed important modifications of the protein dynamics: while glutamine, glutamate, and K603A mutation accelerates subunit exchange, Fru6P and GlcN6P totally prevent it. These results are described in light of the available structural information, providing additional useful data for both the characterization of GImS catalytic process and the design of new GImS inhibitors. Finally, time-resolved noncovalent MS can be proposed as an additional biophysical technique for real-time monitoring of protein dynamics.
\end{abstract}

Key words: Glucosamine-6-phosphate synthase GlmS, Noncovalent mass spectrometry, Subunit kinetic exchange, Protein dynamics, Protein/protein interactions

\section{Introduction}

Glucosamine-6-phosphate synthase (GlmS) is an enzyme U that belongs to the glutamine-dependent amidotrans-

Correspondence to: Sarah Sanglier-Cianférani; e-mail: sarah.cianferani@unistra.fr ferases family. GlmS catalyses the first step of hexosamine metabolism by converting D-fructose-6-phosphate (Fru6P) into D-glucosamine-6-phosphate (GlcN6P) using L-glutamine (Gln) as a nitrogen donor [1]. This enzyme was recently recognized as an interesting pharmacologic target in the complications of diabetes [2].

Received: 3 October 2010

Revised: 28 November 2010

Accepted: 10 December 2010

Published online: 15 January 2011 
GlmS is composed of two independent domains with distinct structures and complementary functions [3]. The $\mathrm{N}$-terminal domain is involved in glutaminase activity whereas the C-terminal part is responsible for the synthase activity (Figure 1). The former catalyses glutamine hydrolysis into glutamate and ammonia, which is then transferred through an internal channel to the later in order to convert bound Fru6P into GlcN6P [4]. Escherichia coli GlmS selfassociates through the synthase domain of both subunits to form a functional dimer, while glutaminase domains are located on the opposite sides of the core dimer [5]. Importantly, Fru6P binding site is formed by the association of both synthase domains, which gives a biological significance to this oligomerization state [4].

The results of X-ray crystallography studies have been recently compiled to provide an overall description of GlmS catalytic mechanism [3, 6, 7]. Different steps were identified during the whole catalytic process. Binding of a sugar in the synthase active site first induces a conformational change that closes the so-called "C-tail" over the synthase site,

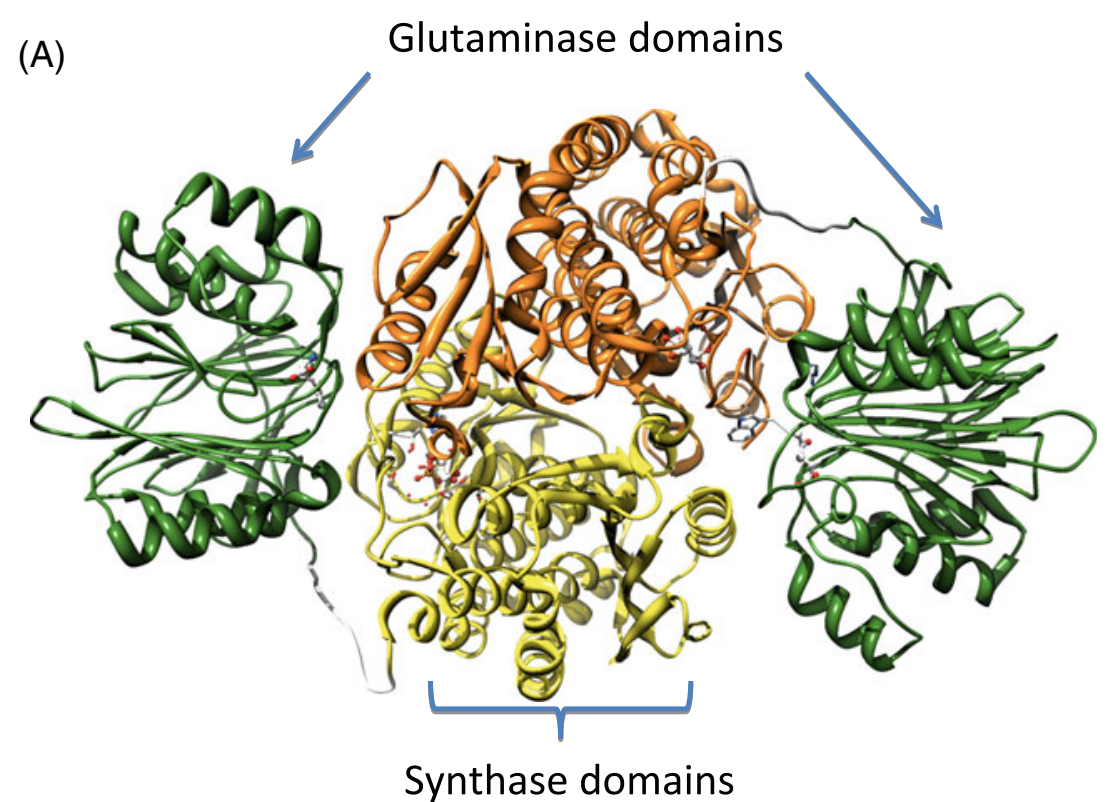

(B)

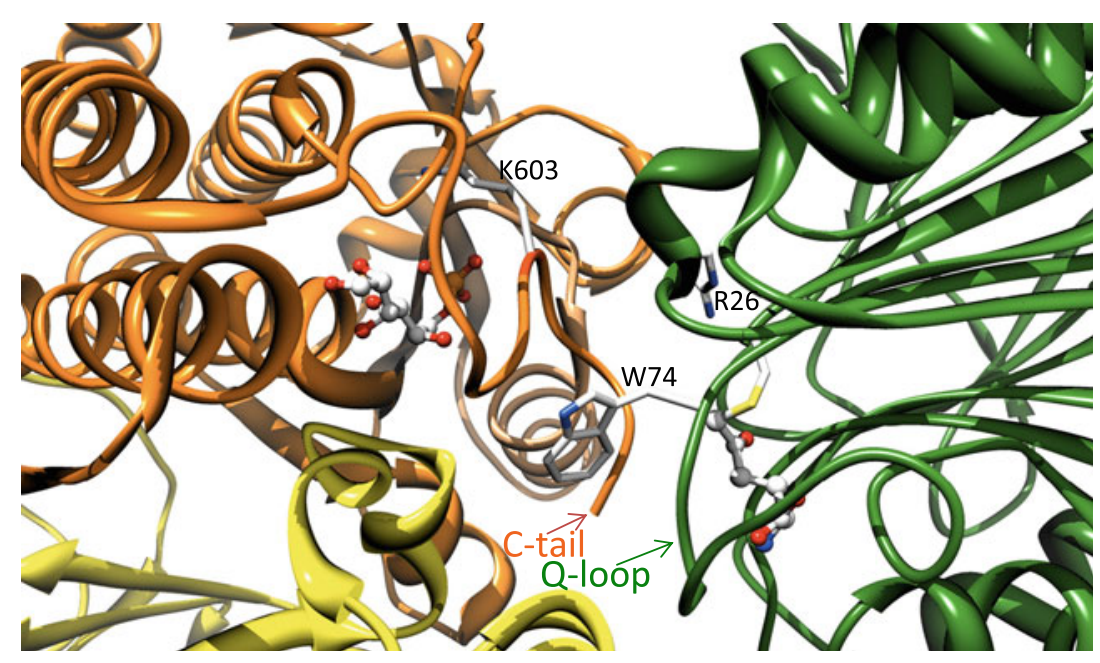

Figure 1. X-ray structure of the Glucosamine-6P-Synthase. Pictures were generated using UCSF Chimera ver. 1.4.1, http:// www.cgl.ucsf.edu/chimera (Protein Data Bank accession numbers 2j6h) [9]. (A) View of the glutaminase and synthase sites with the dimer interface constituted mainly by interaction between two synthase sites of each monomer. (B) Zoom on the catalytic part of one monomer emphasizing the synthase site occupied with Fru6P and the glutaminase site occupied by DON (6-diazo 5-oxo L-norleucine). The two sites, respectively, closed by the C-tail (residues 600-608) and the Q-loop (residues 73-81) are connected by a hydrophobic channel delineated by C-tail, the His-loop of the other subunit (residues $503^{\star}-505^{\star}$ ), Arg 26 and Trp74 
orders the glutaminase domain, and forms a blocked transient channel connecting both domains. Glutamine binding then triggers an additional conformational change, which closes the so-called "Q-loop" over the glutaminase site and opens the ammonia channel through a rotational movement [8]. Ammonia generated in the glutaminase active site can thus be transferred to the synthase active site of the same subunit through the now structured internal channel. Solvent shielding provided by this hydrophobic channel allows ammonia to retain its nucleophilic character by preventing its protonation by water, which would result in a considerable loss of efficiency [4].

This structural description of the catalytic process has been confirmed by kinetic studies to provide a full description of the protein dynamics (for review see [7]). However, to our knowledge, no data has been made available on the dynamics of the dimer formation, although X-ray crystallography has enlightened the importance of such an association to fully assemble synthase domain active site [4]. This question was thus addressed in the present study using time-resolved noncovalent mass spectrometry.

Since the advent of electrospray ionization and the first publications evidencing the possibility to analyze intact noncovalent edifices in the gas phase $[10,11]$, such a use of mass spectrometry has come a long way and is now a wellestablished technique to study protein/protein or protein/ ligand interactions [12-15]. However, while this technique has mainly been used to investigate steady-state protein oligomerization equilibrium, only few studies have reported so far its use to monitor the dynamics of multimeric protein assemblies $[14,16]$. In one of the first studies performed, Vis et al. used nanoESI-MS to monitor the formation of protein heterodimers of HU proteins from Bacillus stearothermophilus and Bacillus subtilis [17]. The mass difference of each dimer was large enough to distinctly observe the formation of the heterodimer. They found that the process of subunit exchange could be fitted by a single exponential function; the rate of heterodimer formation appeared strongly dependent on the salt concentration used for the experiment. Sobott et al. followed the kinetic exchange reaction between subunits of two sHSPs [18]. ESI-MS was used to monitor in real-time the evolution of transient species occurring via the main exchange of dimeric species between pea and wheat HSPs. Investigating the rate of subunit exchange in wild type (WT) $\alpha \mathrm{B}$ - and C-truncated $\alpha \mathrm{A}$ crystalline homo-oligomers [19], Aquilina et al. concluded that the observed reduced kinetic exchange with the $\mathrm{C}$-truncated $\alpha \mathrm{A}$ crystalline comparing to the WT has no effect upon chaperone efficiency. Keetch et al. studied the effect of the L55P single point mutation on the subunits exchange of tetrameric transthyretin, a protein involved in systemic amyloid disease [20]. They used isotopically labeled transthyretin preparations to induce a sufficient mass difference allowing the use of ESI-MS to monitor the formation of heterotetramers. Different subunit exchange dynamic properties were found between WT and L55P forms.

It is noteworthy that a prerequisite to such ESI-MS studies is that compounds generated after subunits exchange display sufficient mass differences so that the different species in equilibrium give rise to non-overlapping signals. To fulfill this requirement, the strategy used in previously described works was to use either homologous proteins from different organisms displaying adequate mass differences or different isotopically labeled protein preparations. In the present work, we used ${ }^{14} \mathrm{~N}$ and ${ }^{15} \mathrm{~N}$ labeled GlmS to generate a light and heavy dimer with enough mass difference to monitor subunit exchange by noncovalent ESI-MS. This approach was first applied to the free enzyme to evaluate the mechanism of reaction and the related kinetic parameters. We then investigated the influence of (1) substrate binding, (2) product binding, and (3) K603A single mutation point, which are all likely to induce modifications of the dimer interface and thus subsequent dynamics of exchange. Results are described in the light of the detailed knowledge of the catalytic mechanism. Finally, the use of time-resolved noncovalent mass spectrometry is discussed in the context of lead discovery/optimization applications when enzymes are targeted.

\section{Experimental}

\section{Purification}

GlmS was overexpressed in recombinant E. coli JM109 harboring a pUC plasmid containing the gImS gene [8]. A $18 \mathrm{~g}$ bacterial pellet was collected from an overnight culture in minimum medium $\left(5 \mathrm{~L}\right.$ fermentor, $37{ }^{\circ} \mathrm{C}$, $200 \mathrm{rpm}$, oxygenation $5 \mathrm{~L} / \mathrm{min}$ ). The protein was purified as previously described [8]. ${ }^{15} \mathrm{~N}$ GlmS was produced from a similar culture where ${ }^{14} \mathrm{NH}_{4} \mathrm{Cl}$ was replaced by ${ }^{15} \mathrm{NH}_{4} \mathrm{Cl}$. Prior to mass spectrometry experiments, nonvolatile salts were removed by exchanging the sample buffer against $50 \mathrm{mM}$ ammonium acetate buffer (Merck, Darmstadt, Germany) ( $\mathrm{pH}$ 6.8). Ammonium acetate enables native structure of proteins to be preserved and is compatible with ESI-MS analysis. A successive series of seven dilution/concentration steps was performed at $4{ }^{\circ} \mathrm{C}$ and $10,000 \mathrm{rpm}$ using $10 \mathrm{kDa}$ PES Vivaspin microconcentrator (Vivascience, Hannover, Germany), with a concentration factor of $10 /$ step. Deionized water $(18.2 \mathrm{M} \Omega)$ was generated from a water filtering device (Millipore, Bedford, USA).

\section{Mass Spectrometry}

Mass spectrometry analyses were performed using an ESITOF mass spectrometer (MicrOTOF; Bruker Daltonics, Bremen, Germany) calibrated using multiply charged ions of horse heart myoglobin (Sigma Aldrich, St. Louis, MO, USA). Samples were continuously infused into the ESI ion 
source at a flow rate of $3 \mu \mathrm{L} / \mathrm{min}$. Drying gas temperature was set at $573 \mathrm{~K}$.

For analysis in denaturing conditions, samples were diluted to $1 \mathrm{pmol} / \mu \mathrm{L}$ in a $1 / 1$ water/acetonitrile mixture ( $\mathrm{vol} / \mathrm{vol}$ ) acidified with $1 \%$ formic acid and standard interface parameters were used to obtain best mass accuracy. In these conditions noncovalent interactions are disrupted, allowing molecular weight measurement of the monomer with a good accuracy (better than $0.01 \%$ ).

For analysis in non-denaturing conditions, samples were directly infused in $50 \mathrm{mM}$ ammonium acetate. Interface parameters were optimized to obtain the best sensitivity and spectrum quality without affecting noncovalent complexes stability. The voltage applied on the capillary exit, named declustering voltage, was set to $250 \mathrm{~V}$, and the pressure in the interface was fixed at 3.2 mbar. Under these conditions, we were able to obtain improved ion desolvation and transmission without inducing dissociation of protein/protein complexes. Pressure and declustering voltage are parameters controlling the energy given to ions in the interface of the micrOTOF mass spectrometer and must be optimized in each case.

\section{Fitting}

Light-, heavy-, and hetero-dimers were considered to have identical ESI response factors as they display similar molecular weights and identical charge state distributions [21]. Heterodimer relative abundances were calculated based on the intensity of the three most intense charge states which are the $24+, 25+$, and $26+$ charge states. As the peak of heterodimer is superimposed with the peak of truncated heavy dimer, heterodimer intensity was corrected for the contribution of truncated heavy dimer assuming that the intensity of this latter species is equal to the intensity of truncated light dimer. All graphs were produced using SigmaPlot 2000, ver. 6.00 (Systat Software Inc., San Jose, CA, USA). All the curves were fitted by a single exponential rise to maximum model: $a \times\left(1-\mathrm{e}^{-\mathrm{bt}}\right)$. The parameter "a" was fixed at $50 \%$ and "b" was the unique variable. Standard errors were calculated by SigmaPlot for a 95\% confidence.

\section{Results and Discussion}

\section{GlmS Subunit Exchange Occurs on Day Time Scale}

The use of mass spectrometry to follow the kinetic exchange of subunits interacting in a macromolecular complex is a general approach described by Sharon and Robinson [14]. Two critical conditions must be fulfilled: (1) a sufficient mass difference must be observed, and (2) the exchange must be in a time frame compatible with mass spectrometry techniques. The mass difference can be obtained by working with different protein isoforms or by using different isotopically labeled proteins. In the present work, GlmS dimers were overproduced both in ${ }^{14} \mathrm{~N}$ and ${ }^{15} \mathrm{~N}$ media. This approach has also been recently used by Natan et al. to measure the composition and rate of $\mathrm{p} 53$ tetramers formation in presence or absence of its DNA response element [22]. In case of GlmS, ESI mass measurements under denaturing conditions reveal molecular weights of $66765 \pm 2 \mathrm{Da}$ and $67554 \pm 2$ Da for ${ }^{14} \mathrm{~N}$ GlmS and ${ }^{15} \mathrm{~N}$ GlmS, respectively (Figures 2a and b). The mass difference of 789 Da corresponds to a ${ }^{15} \mathrm{~N}$ incorporation rate of around $95 \%$. It is also worth noting that the mass measured for ${ }^{14} \mathrm{~N}$ GlmS is in agreement with the expected molecular weight of the protein without its N-terminal methionine $(66763 \mathrm{Da})$ as previously confirmed by Edman sequencing [5]. Typical ESI mass spectra recorded in non-denaturing conditions, displaying peaks with high $\mathrm{m} / \mathrm{z}$ ratios and narrow charge state distributions, are presented in Figures $2 \mathrm{c}$ and d. No species corresponding to any light or heavy monomeric GlmS forms are detected. The measured masses of $133658 \pm 15 \mathrm{Da}$ and $135272 \pm 14 \mathrm{Da}$ correspond, respectively, to light $\left({ }^{14} \mathrm{~N}\right)$ and heavy $\left({ }^{15} \mathrm{~N}\right) \mathrm{GlmS}$ dimers, indicating thus that experimental conditions allow weak noncovalent interactions between monomers to be preserved in the gas phase of the mass spectrometer. Moreover, the observed mass difference of about $1600 \mathrm{Da}$ between the light and the heavy dimer in conjunction with the close charge state distribution at high $\mathrm{m} / \mathrm{z}$ ratios obtained in non-denaturing conditions is sufficient to distinguish light, heavy, and mixed dimers.

In a first experiment, both proteins were incubated in equimolar concentrations at $4{ }^{\circ} \mathrm{C}$. However, after more than 2 days of incubation, no signal corresponding to heterodimer could be detected. In order to increase the exchange rate, incubation temperature was raised to $37{ }^{\circ} \mathrm{C}$. As expected, results showed a faster subunit exchange at this temperature (data not shown); therefore, subsequent experiments were all performed at $37{ }^{\circ} \mathrm{C}$. Figure 3 represents ESI mass spectra recorded under non-denaturing conditions and instrumental settings optimized for intact noncovalent GlmS dimer transmission (see the Experimental section). Light $\left({ }^{14} \mathrm{~N}\right)$ and heavy $\left({ }^{15} \mathrm{~N}\right)$ GlmS dimers were mixed in equimolar amounts, and subunit exchange kinetics between $\left({ }^{14} \mathrm{~N}\right)$ and $\left({ }^{15} \mathrm{~N}\right) \mathrm{GlmS}$ was monitored over a 2-d period (Figure 3). The indication of subunit exchange is easily apparent after $8 \mathrm{~h}$ of reaction (Figure 3a). Indeed, while the species with the highest intensities remain the ${ }^{14} \mathrm{~N} /{ }^{14} \mathrm{~N}$ and ${ }^{15} \mathrm{~N} /{ }^{15} \mathrm{~N}$ homodimers, an additional species is detected with a molecular weight of $134465 \pm 10 \mathrm{Da}$ corresponding to the ${ }^{14} \mathrm{~N} /{ }^{15} \mathrm{~N}$ heterodimer (theoretical MW=134325 Da). Monitoring this reaction over longer incubation periods reveals an increase of the heterodimer proportion (Figures $3 b$ and c). Equilibrium distribution is still not observed after $51 \mathrm{~h}$ in these experimental conditions, demonstrating, thus, a slow exchange kinetics.

The subunit exchange time frame is dependent of the reactivity of the species; it is an experimental parameter that can be hardly modulated in order to match MS requirements. For reactions occurring within seconds or less, special instrumental setups have been designed based on the mixing 

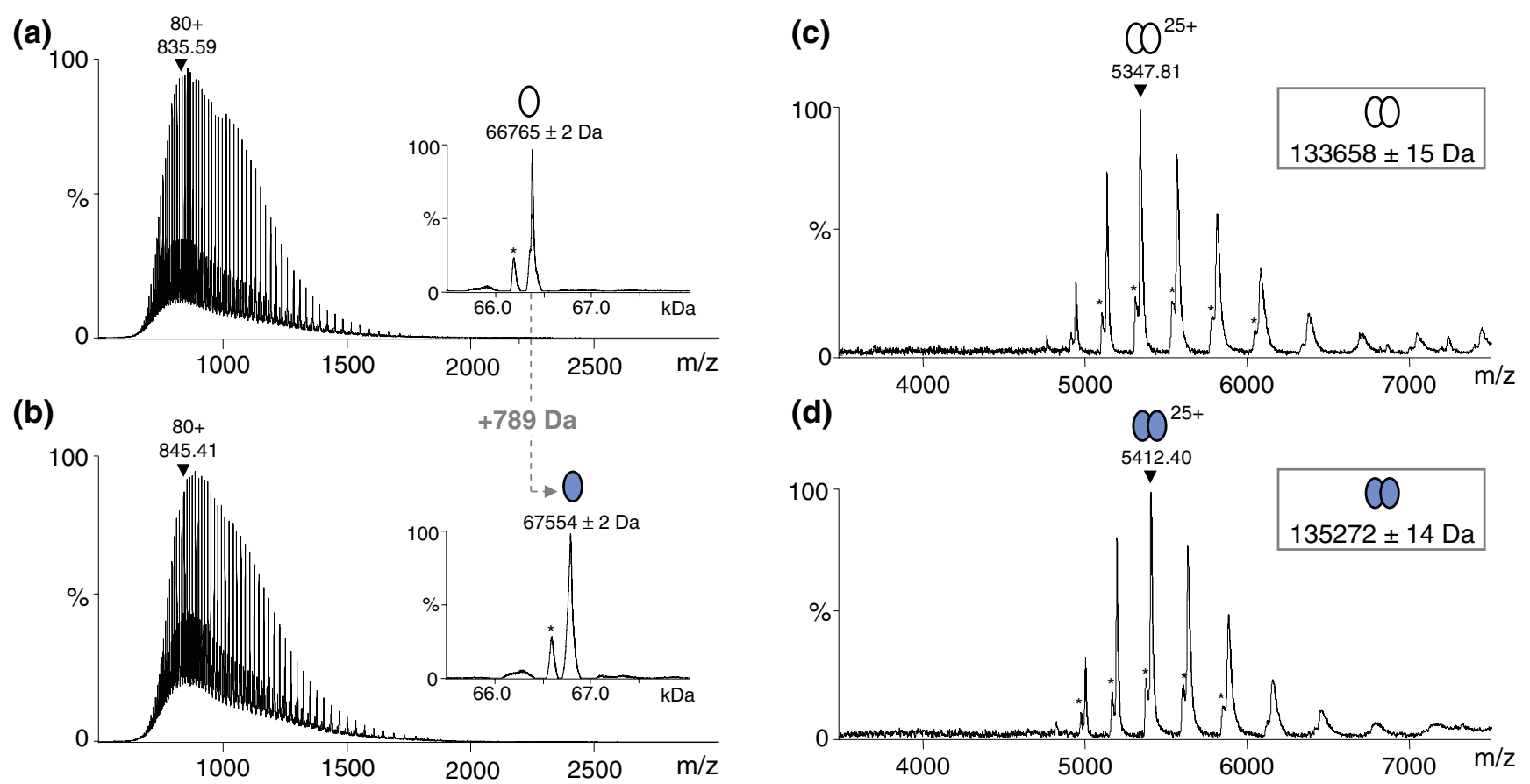

Figure 2. ESI-MS analyses of ${ }^{14} \mathrm{~N}$ and ${ }^{15} \mathrm{~N}$ GImS. (a) ${ }^{14} \mathrm{~N} \mathrm{GImS}$ and (b) ${ }^{15} \mathrm{~N} \mathrm{GlmS}$ were diluted to $1 \mu \mathrm{M}$ in a $1: 1$ water:acetonitrile mixture containing $1 \%$ formic acid. Inserts correspond to MaxEnt deconvolutions. (c) ${ }^{14} \mathrm{~N}$ GlmS and (d) ${ }^{15} \mathrm{~N}$ GlmS were diluted to $2 \mu \mathrm{M}$ in $50 \mathrm{mM}$ ammonium acetate buffer. White and blue circles are related to ${ }^{14} \mathrm{~N}$ and ${ }^{15} \mathrm{~N}$ GlmS, respectively. ${ }^{*}$ Corresponds to a GlmS degradation product (loss of the N-terminal CGIV peptide)

of the species before MS measurement [23-26]. In our case, the subunit exchange was extremely slow: subunit exchange was observed at $37{ }^{\circ} \mathrm{C}$ during more than 2 days without apparent degradation or loss of enzyme stability. Various rate constants were reported in the literature. In the case of HSP, Sobott et al. described "fast" dissociation rates occurring in minutes [18], whereas similar slow interconversion, on the day time scale, was observed by Vis et al. on HU proteins [17]. These authors rationalize their observations by the mechanism of heterodimer formation: homodimer may first dissociate into individual folded monomers, which subsequently re-associate to form heterodimeric species. No direct relation between the observed exchange kinetics and the thermodynamic dissociation constant of the complex subunits could be established. However, if the dissociation of the dimer into monomers is the rate limiting step, it could be assumed that faster exchange kinetics could be the result of a destabilized dimer interface.

\section{Subunit Exchange Dynamics is Tuned by Ligand-Binding}

To investigate the influence of GlmS substrates and reaction products on monomer exchange, similar ESI-MS experiments were performed in presence of Fru6P, GlcN6P, Gln, or Glu, respectively. Interestingly, the exchange rate is strongly affected by the presence of either sugars or amino acids.

In contrast with the free enzyme, the presence of a 10fold molar excess of Fru6P or GlcN6P $\left(10 \mu \mathrm{M}{ }^{14} \mathrm{~N}+10 \mu \mathrm{M}\right.$ ${ }^{15} \mathrm{~N}$ GlmS dimer $+100 \mu \mathrm{M}$ Fru6P or GlcN6P), abolished subunit interconversion at least on a 2-d time scale (Figure 3d). These observations demonstrate that the subunit exchange reaction is drastically reduced, which suggests that dissociation of the dimer subunits could be the limiting step of the exchange reaction.

These results suggest that sugar-binding induces a structural rearrangement enhancing the stability of the dimer interface and blocking monomer dissociation. Conformational changes of GlmS induced upon sugar binding were previously studied using X-ray crystallography [27]. Comparison of GlmS, GlmS-Fru6P, and GlmS-GlcN6P crystallographic structures indicated that the $\mathrm{C}$-terminal part of the synthase domain (C-tail) becomes ordered upon sugar binding and covers the adjacent synthase site. In the closed conformation, the $\mathrm{C}$-tail of one monomer interacts with the sugar at the synthase site as well as the glutaminase domain of the other monomer and has been shown to form the major part of the ammonia channel that connects the glutaminase and synthase domains. This rearrangement should strengthen the dimer interface as it induces additional interactions between the glutaminase and synthase domains of one monomer with the synthase domain of the other one. Recent microcalorimetry experiments provided additional evidence for strong enzyme stabilization by Fru6P since a $7{ }^{\circ} \mathrm{C}$ shift in $\mathrm{T}_{\mathrm{m}}$ corresponding to a $120 \mathrm{kcal} \mathrm{mol}^{-1}$ increase in enthalpy was observed [28]. The experimental ESI-MS data obtained by monitoring the heterodimer formation in presence of sugar-binding are in good agreement with these conformational changes. 


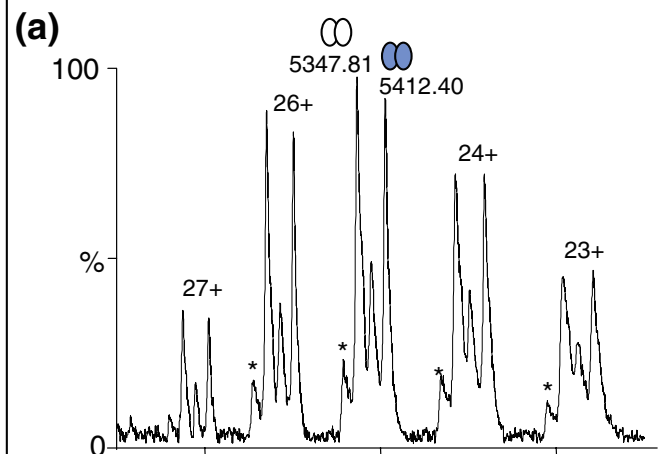

(b)

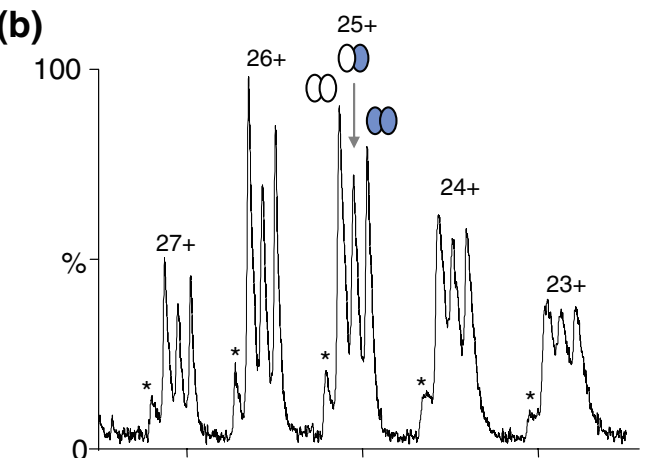

(c)

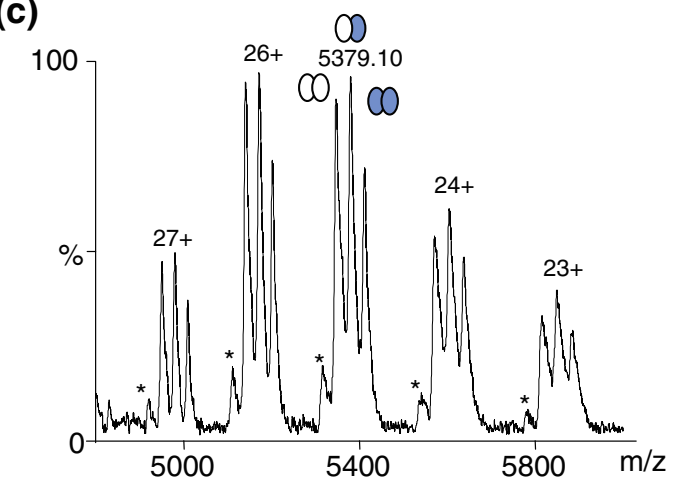

(d)

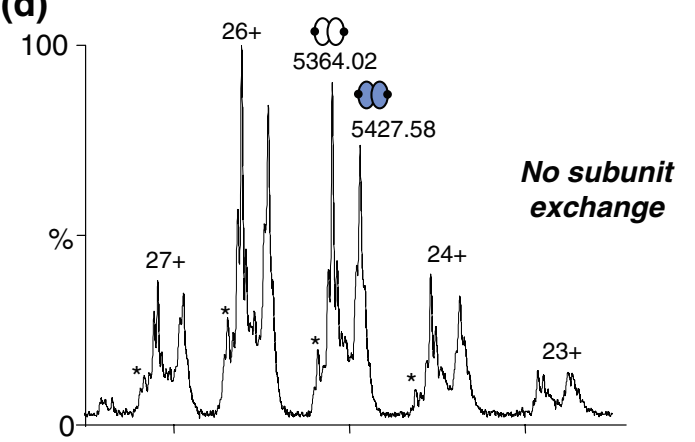

(e)

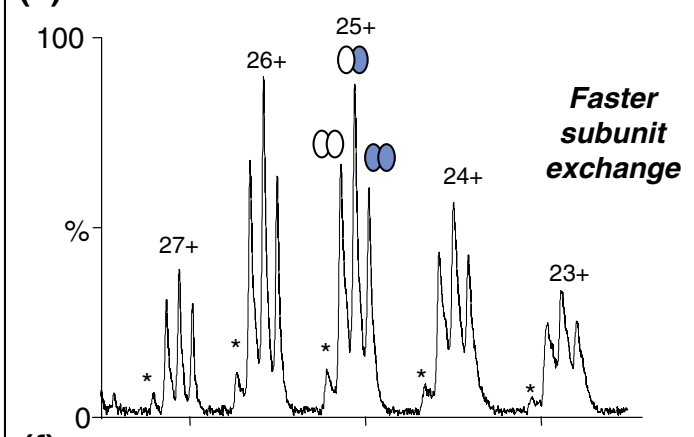

(f)

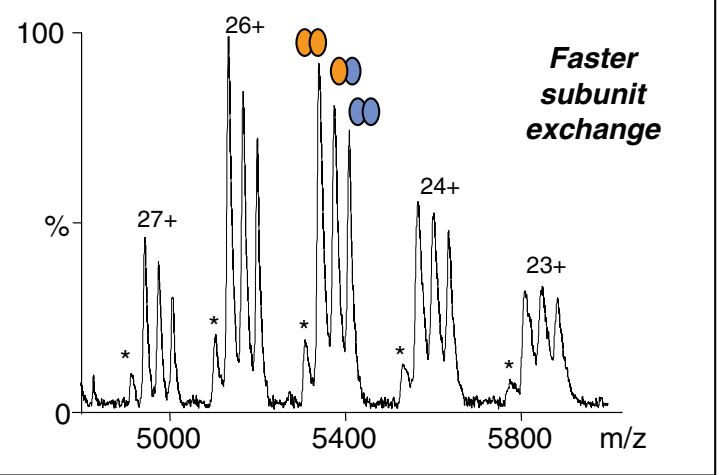

$\begin{array}{ll}\text { CO } & { }^{14} \mathrm{~N} /{ }^{14} \mathrm{~N}+2 \text { Fru6P }(134079 \pm 7 \mathrm{Da}) \\ \text { CO } & { }^{15} \mathrm{~N} /{ }^{15} \mathrm{~N}+2 \text { Fru6P }(135664 \pm 9 \mathrm{Da}) \\ C & { }^{14} \mathrm{~N}(\mathrm{~K} 603 \mathrm{~A}) /{ }^{14} \mathrm{~N}(\mathrm{~K} 603 \mathrm{~A})(133535 \pm 12 \mathrm{Da}) \\ \infty & { }^{14} \mathrm{~N}(\mathrm{~K} 603 \mathrm{~A}) /{ }^{15} \mathrm{~N}(134400 \pm 19 \mathrm{Da})\end{array}$

Figure 3. Monitoring of GImS subunit exchange by ESI-MS in non-denaturing conditions. (a) $-(\mathbf{c}){ }^{14} \mathrm{~N}$ and ${ }^{15} \mathrm{~N}$ GImS $(2 \mu \mathrm{M}$, each) were incubated at $37{ }^{\circ} \mathrm{C}$ for (a) $8 \mathrm{~h}$, (b) $20 \mathrm{~h}$, and (c) $51 \mathrm{~h}$. (d) ${ }^{14} \mathrm{~N}$ and ${ }^{15} \mathrm{~N} \mathrm{GlmS}(10 \mu \mathrm{M}$, each) were incubated in presence of $100 \mu \mathrm{M}$ Fru6P for $2 \mathrm{~d}$ at $37^{\circ} \mathrm{C}$. (e) ${ }^{14} \mathrm{~N}$ and ${ }^{15} \mathrm{~N} \mathrm{GlmS}\left(2 \mu \mathrm{M}\right.$, each) were incubated in presence of $100 \mu \mathrm{M} \mathrm{Gln}$ for $20 \mathrm{~h}$ at $37^{\circ} \mathrm{C}$. (f) ${ }^{14} \mathrm{~N}(\mathrm{~K} 603 \mathrm{~A})$ and ${ }^{15} \mathrm{~N} \mathrm{GlmS}\left(2 \mu \mathrm{M}\right.$, each) were incubated at $37^{\circ} \mathrm{C}$ for $20 \mathrm{~h}$. ${ }^{*}$ Corresponds to a GlmS degradation product (loss of the $\mathrm{N}$-terminal CGIV peptide)

In the presence of an excess glutamine or glutamate, the monomer exchange also appeared to be modified. Several incubations performed at increasing substrate concentrations of $0,5,10$, and $100 \mu \mathrm{M}$ for a total dimer concentration of $4 \mu \mathrm{M}\left({ }^{14} \mathrm{~N}+{ }^{15} \mathrm{~N}\right.$ GlmS $)$ resulted in faster heterodimer formation (Figure 3e). Neither Gln nor Glu adduct was observed, even at the highest ligand concentration used. This most likely arises from gas-phase dissociation of protein/ ligand complexes provoked by the use of high declustering voltages, which were necessary to reach optimal desolvation and transmission of GlmS dimer ions (see Experimental part).In this case, investigating the kinetic exchange of subunits allowed us to enlighten the ligand effect using mass spectrometry without binding observation. The time dependence of heterodimer formation is represented by plotting the percentage of heterodimer directly deduced from peak intensities observed on ESI mass spectra as a function of time (Figure 4). All curves obtained were fitted to a single- 
exponential function to extract a first-order dissociation rate constant for GlmS dimer in the chosen conditions. This assumption is in agreement with the few publications that have reported multiprotein subunit exchanges in the literature and that assumed that, in the case of a dimer, the kinetic exchange could be fitted by a single-exponential function $[17,18]$. This means that the dissociation of subunits is the limiting step in contrast to the monomer association that is considered as a rapid event. Heterodimer formation is then considered as a first order kinetic reaction. We also assumed that all species (light-, heavy-, and heterodimers) display similar chemical reactivities and ESI response factors. These assumptions prompted us to use a fixed maximum at $50 \%$ for the heterodimer relative abundance and only one free parameter in our model was fitted to the experimental data (see Material and Methods). The calculated rate constants for subunit exchanges were $0.034 \pm 0.005 \mathrm{~h}^{-1}$ for the free enzyme and $0.043 \pm 0.006 \mathrm{~h}^{-1}$, $0.056 \pm 0.004 \mathrm{~h}^{-1}, 0.090 \pm 0.005 \mathrm{~h}^{-1}$ for an addition of 5,10 , $100 \mu \mathrm{M}$ of glutamine or glutamate, respectively (Figures $4 \mathrm{a}-$ d). The average experimental error obtained on the fixed parameter " $\mathrm{a}=50$ " was about 2 , suggesting that our experimental data suitably fitted the model and thus confirmed the theoretical assumptions regarding chemical reactivities and ESI response factors. Finally the calculated rate constant for subunit exchange increased as a function of ligand concentration. For the highest ligand concentration tested $(100 \mu \mathrm{M})$, the rate constant increases up to three times in comparison to the one obtained under initial conditions without ligand addition.

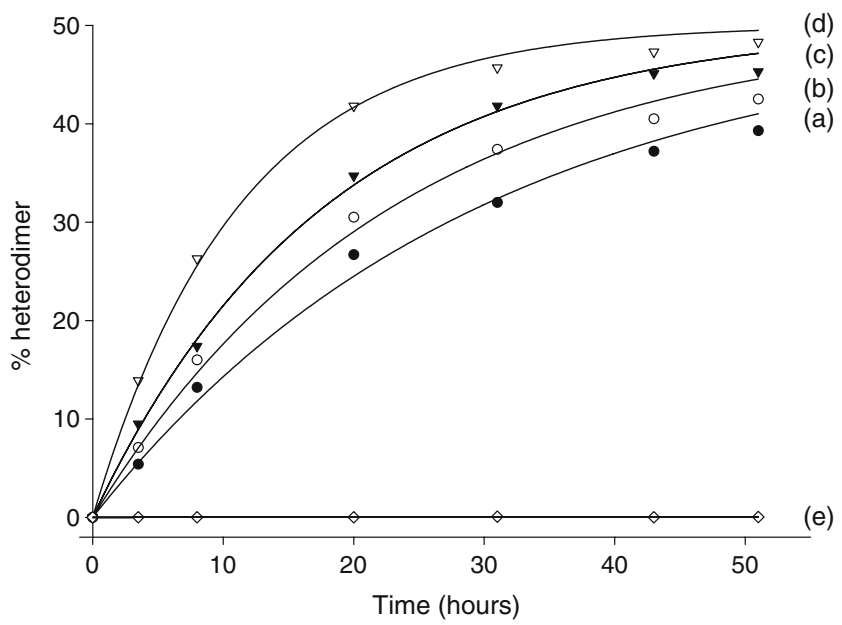

Figure 4. Effect of substrate and product binding on the subunit exchange rate. ${ }^{14} \mathrm{~N}$ GlmS $(2 \mu \mathrm{M})$ and ${ }^{15} \mathrm{~N}$ GImS $(2 \mu \mathrm{M})$ were incubated at $37{ }^{\circ} \mathrm{C}$ (a) alone or in presence of, (b) $5 \mu \mathrm{M}$ glutamine or glutamate, (c) $10 \mu \mathrm{M}$ glutamine or glutamate, (d) $100 \mu \mathrm{M}$ glutamine or glutamate, $(\mathrm{e}){ }^{14} \mathrm{~N}$ GlmS $(10 \mu \mathrm{M})$ and ${ }^{15} \mathrm{~N}$ GImS $(10 \mu \mathrm{M})+100 \mu \mathrm{M}$ Fru6P or GlcN6P. The percentage of ${ }^{14} \mathrm{~N} /{ }^{15} \mathrm{~N}$ GlmS heterodimer was plotted versus the incubation time. All curves were fitted to a single-exponential function
The fact that glutamine or glutamate affects the dynamics of subunit exchange in this experiment suggests that those ligands trigger a conformational change that destabilizes the dimer interface. It was found by X-ray crystallography that glutamine binding triggers the closure of the glutaminase site by a flexible loop from the glutaminase domain called the "Q-loop" and opens the ammonia channel between both glutaminase and synthase sites [8]. This closure was followed by a hinged movement of $21^{\circ}$ between the glutaminase domains to prevent the destabilization of the dimer interface involving strongly the Q-loop. The faster kinetic exchange observed by MS in presence of glutamine could be induced by this latter movement that opens the ammonia channel apparently at the expense of some interactions at the binding interface of the dimer.

Experimental data obtained by monitoring subunit exchange using MS support the observations based on X-ray crystallography in so far that both sugars and glutamine derivatives trigger a conformational change of the enzyme that affects the dimer interface by inducing an ammonia channel connecting two active sites. These structural changes allow explaining the overall catalytic mechanism of the enzyme.

\section{Destabilization of the Dimer Interface by Amino Acid Mutation}

Finally, we evaluated this noncovalent MS-based approach to investigate the influence of GlmS single point mutation on GlmS subunit exchange. Mixing equal amounts of the mutant K603A and heavy wild type GlmS protein (as the heavy form of the mutated protein was not available) resulted in subunit exchange with an equilibrium reached

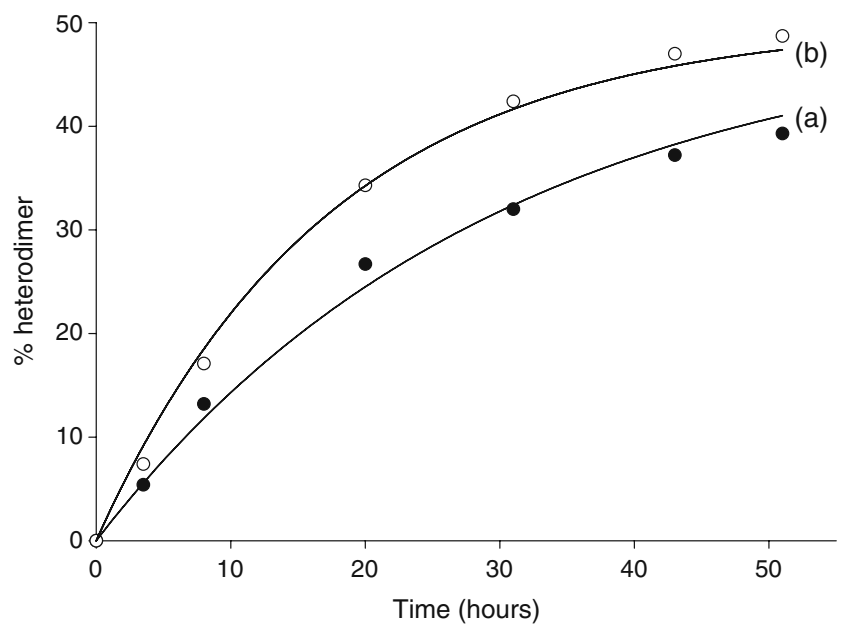

Figure 5. Effect of a single point mutation (K603A) at the dimer interface on the subunit exchange rate. ${ }^{15} \mathrm{~N}$ GlmS $(2 \mu \mathrm{M})$ was incubated at $37{ }^{\circ} \mathrm{C}$ in presence of a) ${ }^{14} \mathrm{~N}$ GlmS $(2 \mu \mathrm{M})$ and $\mathbf{b}){ }^{14} \mathrm{~N}(\mathrm{~K} 603) \mathrm{GlmS}(2 \mu \mathrm{M})$. The percentage of ${ }^{14} \mathrm{~N}$ $(\mathrm{K} 603) /{ }^{15} \mathrm{~N}$ GlmS heterodimer was plotted versus the incubation time. Both curves were fitted to a single-exponential function 
for a maximum at around $50 \%$ after $51 \mathrm{~h}$ incubation (Figure 5). The calculated rate constant for subunit exchange of $0.058 \pm 0.005 \mathrm{~h}^{-1}$ is superior to the one obtained with the wild type GlmS protein $\left(0.034 \pm 0.005 \mathrm{~h}^{-1}\right)$. The fact that the K603A mutation accelerates GlmS subunit exchange would suggest that this mutation destabilizes the dimer interface (compare Figures $3 b$ and $\mathrm{f}$ ).

From this experiment, it could be concluded that noncovalent MS can be used to evidence the effect induced by a point mutation and can serve, in combination with X-ray diffraction, as a discriminating tool for a better comprehension of the overall GlmS catalytic reaction. Indeed, alteration of function is not necessarily correlated with disruption of active site or ligand-binding site. Most proteins have mobile parts and the introduction of a point mutation into any part of the protein can be detrimental to activity if the latter relies on the propagation of a conformational change. A preliminary work reported that K603R mutation induces a 40-fold decrease of GlmS synthase activity, evidencing thus the importance of this residue for the C-terminal domain activity [29]. Here we used the K603A mutant which induces a 24,000 -fold decrease in enzyme activity. When the light mutant K603A was mixed with the heavy wild type (WT) protein in the absence of any substrate, we observed a 1.7fold acceleration of subunit exchange compared to the wild type protein. These data provide strong evidence that this mutation destabilizes the dimer interface.

\section{Conclusions}

In the present work, the potentialities of noncovalent MS for the characterization of systems out of equilibrium have been evaluated. We developed an ESI-MS-based experimental approach that afforded us to follow in real-time the subunit exchange dynamics of GlmS dimer. Rates of subunit exchange have been deduced from several experiments in which different parameters were tested such as the presence of various ligands or the mutation of one amino acid. In particular, it has been demonstrated that extracted rate constants for subunit exchange depend on the presence of effector molecules that interact with the protein and also from the amino acid sequence of the protein itself, enlightening the crucial role of specific residues. The binding of sugars (Fru6P and GlcN6P) was found to stop subunit exchange. This is in agreement with X-ray crystallography data reporting a close conformation upon sugar binding where the $\mathrm{C}$-tail of one monomer interacts with the sugar and the glutaminase/synthase domains of the other monomer, enhancing, thus, the dimer stability. The exchange in presence of an excess of Gln/Glu displays a significant increased rate that could be explained by the closure of a flexible loop of the glutaminase domain (Qloop) that destabilizes the dimer interface. Results obtained with the GlmS system will help (1) to get new insights into the comprehension of the catalytic process of glutamine- dependent amidotransferases, (2) to guide the search for new GlmS inhibitors.

We also demonstrated that noncovalent MS can now be proposed as a supporting technique for the design of dimerization inhibitors. Indeed, this MS approach is now a well-established technique for the characterization of protein/ligand complexes. Using well-adapted analytical strategies, it is possible to gain insights into the binding stoichiometry of such complexes, their affinity, and their specificity, as well as the nature of noncovalent interactions involved between a ligand and a target protein (electrostatic versus hydrophobic), providing, thus, crucial information in the context of drug design [30-32]. In the present study, we showed that noncovalent MS could not only be used to evaluate how tightly a molecule binds to a target protein but also to evidence the structural effect of such a binding on the overall stability of a protein/protein complex. As the development of ligands able to alter protein/protein interactions represents one of the future challenges in drug discovery [33], the present methodology could provide strong support for the rational design of new lead molecules targeting protein/protein contacts. For instance, the strategy described in the present paper for the study of the dimeric $E$. coli $\mathrm{GlmS}$ could be extended to tetrameric eukaryotic GlmS. In fact, X-ray crystallographic structure shows that GlmS from Candida albicans is a dimer of tight dimers [34]. The different protein/protein interactions involved in this protein architecture could potentially give rise to a different subunit exchange dynamics as that observed for $E$. coli. Given the implication of human GlmS in type 2 diabetes, such a study could provide a strong support for the design of new inhibitors. This approach could then be extended to all proteins available for biophysical characterization, assuming that ${ }^{14} \mathrm{~N}$ and ${ }^{15} \mathrm{~N}$ proteins give similar ESI responses and enough mass difference to be detected by the mass spectrometer. Noncovalent MS could finally be generally used in overall investigation of association/dissociation of biomolecules in solution.

\section{Acknowledgments}

The authors acknowledge funding for this work by the CNRS, the University of Strasbourg UdS and the Region Alsace. G.C. acknowledges the CNRS and Sanofi-Aventis for financial support. C.A. was supported by a studentship from the French Ministère de la Recherche.

\section{References}

1. Ghosh, S., Blumenthal, H.J., Davidson, E., Roseman, S.: Glucosamine Metabolism. V. Enzymatic Synthesis of Glucosamine 6-Phosphate. J Biol Chem 235, 1265-1273 (1960)

2. Buse, M.G.: Hexosamines, Insulin Resistance, and the Complications of Diabetes: Current status. Am J Physiol Endocrinol Metab 290(1), E1E8 (2006)

3. Teplyakov, A., Leriche, C., Obmolova, G., Badet, B., Badet-Denisot, M.A.: From Lobry de Bruyn to enzyme-catalyzed ammonia channeling: Molecular Studies of D-Glucosamine-6P Synthase. Nat Prod Rep 19(1), 60-69 (2002) 
4. Teplyakov, A., Obmolova, G., Badet, B., Badet-Denisot, M.A.: Channeling of ammonia in glucosamine-6-phosphate synthase. $J \mathrm{Mol}$ Biol 313(5), 1093-1102 (2001)

5. Badet, B., Vermoote, P., Haumont, P.Y., Lederer, F., LeGoffic, F.: Glucosamine synthetase from Escherichia coli: Purification, properties, and glutamine-utilizing site location. Biochemistry 26(7), 1940-1948 (1987)

6. Milewski, S.: Glucosamine-6-phosphate synthase-the multi-facets enzyme. Biochim Biophys Acta 1597(2), 173-192 (2002)

7. Durand, P., Golinelli-Pimpaneau, B., Mouilleron, S., Badet, B., BadetDenisot, M.A.: Highlights of glucosamine-6P synthase catalysis. Arch Biochem Biophys 474, 302-317 (2008)

8. Mouilleron, S., Badet-Denisot, M.A., Golinelli-Pimpaneau, B.: Glutamine Binding Opens the Ammonia Channel and Activates Glucosamine-6P Synthase. J Biol Chem 281(7), 4404-4412 (2006)

9. Pettersen, E.F., Goddard, T.D., Huang, C.C., Couch, G.S., Greenblatt, D.M., Meng, E.C., Ferrin, T.E.: UCSF Chimera - a visualization system for exploratory research and analysis. J Comput Chem 25(13), 1605 1612 (2004)

10. Ganem, B., Li, Y.T., Henion, J.D.: Detection of noncovalent receptorligand complexes by mass spectrometry. J Am Chem Soc 113, 62946296 (1991)

11. Katta, V., Chait, B.T.: Observation of the heme-globin complex in native myoglobin by electrospray-ionization mass spectrometry. $\mathrm{J} \mathrm{Am}$ Chem Soc 113, 8534-8535 (1991)

12. Loo, J.A.: Electrospray ionization mass spectrometry: A technology for studying noncovalent macromolecular complexes. Int J Mass Spectrom 200, 175-186 (2000)

13. Heck, A.J., Van Den Heuvel, R.H.: Investigation of intact protein complexes by mass spectrometry. Mass Spectrom Rev 23, 368-389 (2004)

14. Sharon, M., Robinson, C.V.: The role of mass spectrometry in structure elucidation of dynamic protein complexes. Annu Rev Biochem 76, $167-$ 193 (2007)

15. Atmanene, C., Wagner-Rousset, E., Malissard, M., Chol, B., Robert, A., Corvaia, N., Dorsselaer, A.V., Beck, A.: Extending Mass Spectrometry Contribution to Therapeutic Monoclonal Antibody Lead Optimization: Characterization of Immune Complexes Using Noncovalent ESI-MS. Anal Chem 81(15), 6364-6373 (2009)

16. Painter, A.J., Jaya, N., Basha, E., Vierling, E., Robinson, C.V., Benesch, J. L.: Real-time monitoring of protein complexes reveals their quaternary organization and dynamics. Chem Biol 15(3), 246-253 (2008)

17. Vis, H., Dobson, C.M., Robinson, C.V.: Selective association of protein molecules followed by mass spectrometry. Protein Sci 8(6), 1368-1370 (1999)

18. Sobott, F., Benesch, J.L., Vierling, E., Robinson, C.V.: Subunit exchange of multimeric protein complexes. Real-time monitoring of subunit exchange between small heat shock proteins by using electrospray mass spectrometry. J Biol Chem 277(41), 38921-38929 (2002)

19. Aquilina, J.A., Benesch, J.L., Ding, L.L., Yaron, O., Horwitz, J., Robinson, C.V.: Subunit exchange of polydisperse proteins: Mass spectrometry reveals consequences of $\longmapsto$ A-crystallin truncation. $J$ Biol Chem 280, 1485-1491 (2005)

20. Keetch, C.A., Bromley, E.H., McCammon, M.G., Wang, N., Christodoulou, J., Robinson, C.V.: L55P transthyretin accelerates subunit exchange and leads to rapid formation of hybrid tetramers. J Biol Chem 280(50), 4166741674 (2005)

21. Peschke, M., Verkerk, U.H., Kebarle, P.: Features of the ESI mechanism that affect the observation of multiply charged noncovalent protein complexes and the determination of the association constant by the titration method. J Am Soc Mass Spectrom 15, 1424-1434 (2004)

22. Natan, E., Hirschberg, D., Morgner, N., Robinson, C.V., Fersht, A.R.: Ultraslow oligomerization equilibria of p53 and its implications. Proc Natl Acad Sci USA 106(34), 14327-14332 (2009)

23. Kolakowski, B.M., Simmons, D.A., Konermann, L.: Stopped-flow electrospray ionization mass spectrometry: A new method for studying chemical reaction kinetics in solution. Rapid Commun Mass Spectrom 14(9), 772-776 (2000)

24. Wilson, D.J., Konermann, L.: A capillary mixer with adjustable reaction chamber volume for millisecond time-resolved studies by electrospray mass spectrometry. Anal Chem 75(23), 6408-6414 (2003)

25. Simmons, D.A., Wilson, D.J., Lajoie, G.A., Doherty-Kirby, A., Konermann, L.: Subunit disassembly and unfolding kinetics of hemoglobin studied by time-resolved electrospray mass spectrometry. Biochemistry 43(46), 14792-14801 (2004)

26. Attwood, P.V., Geeves, M.A.: Kinetics of an enzyme-catalyzed reaction measured by electrospray ionization mass spectrometry using a simple rapid mixing attachment. Anal Biochem 334(2), 382-389 (2004)

27. Mouilleron, S., Badet-Denisot, M.A., Golinelli-Pimpaneau, B.: Ordering of C-terminal loop and glutaminase domains of glucosamine-6phosphate synthase promotes sugar ring opening and formation of the ammonia channel. J Mol Biol 377(4), 1174-1185 (2008)

28. Valerio-Lepiniec, M., Aumont-Nicaise, M., Roux, C., Raynal, B., England, P., Badet, B., Badet-Denisot, M.A., Desmadril, M.: Analysis of the Escherichia coli glucosamine-6-phosphate synthase activity by isothermal titration calorimetry and differential scanning calorimetry. Arch. Biochem. Biophys. 498(2), 95-104.

29. Badet-Denisot, M.A., Rene, L., Badet, B.: Mechanistic investigations on glucosamine-6-phosphate synthase. Bull Soc Chim Fr 130, 249-255 (1993)

30. Darmanin, C., Chevreux, G., Potier, N., Van Dorsselaer, A., Hazemann, I., Podjarny, A., El-Kabbani, O.: Probing the ultra-high resolution structure of aldose reductase with molecular modeling and noncovalent mass spectrometry. Bioorg Med Chem 12(14), 3797-3806 (2004)

31. Sanglier, S., Atmanene, C., Chevreux, G., Van Dorsselaer, A.: Nondenaturing mass spectrometry to study noncovalent protein/protein and protein/ligand complexes: Technical aspects and application to the determination of binding stoichiometries. Meth Mol Biol 484, 217-243 (2008)

32. Vivat Hannah, V., Atmanene, C., Zeyer, D., Van Dorsselaer, A., Sanglier-Cianférani, S.: Native mass spectrometry: An "ESI" way to support structure- and fragment-based drug discovery. Future Med Chem 2(1), 35-50 (2010)

33. Zinzalla, G., Thurston, D.E.: Targeting protein-protein interactions for therapeutic intervention: A challenge for the future. Future Med Chem 1 (1), 65-93 (2009)

34. Raczynska, J., Olchowy, J., Konariev, P.V., Svergun, D.I., Milewski, S., Rypniewski, W.: The crystal and solution studies of glucosamine-6phosphate synthase from Candida albicans. J Mol Biol 372(3), 672-688 (2007) 